\title{
ORBITAL RADIAL VELOCITY CURVES OF SYMBIOTIC STARS
}

\author{
Michael R. Garcia and Scott J. Kenyon \\ Harvard/Smithsonian Center for Astrophysics, \\ 60 Garden St., Cambridge, MA., USA
}

\begin{abstract}
For the past 6 years we have been measuring the radial velocity of the Mgiant component of Symbiotics Stars with high dispersion echelle spectrographs. Because these velocities are based on the absorption line spectrum of the M-star, very reliable orbital radial velocity curves can be constructed. The orbital solutions derived from these velocity curves allow the approximate size of the binary to be determined, and also provide insight into the origin of the photometric variations seen in these stars. For most of the stars, the photometric variations are due to eclipse or reflection effects (two notable exceptions are $Z$ And and RW Hya). Stars which may be near to filling their Roche lobes are TX CVn, Z And, EG And, UV Aur, and CI Cyg. Stars which do not appear to fill their Roche lobes are AG Dra, RW Hya, and AG Peg.
\end{abstract}

\section{INTRODUCTION}

As our understanding of interacting binary stars is based largely on measurements of stellar masses, periods, and mass transfer rates (see, for example, the study of cataclysmic variables by Patterson 1984), measurements of these most basic parameters for symbiotic stars would seem to be a prerequisite to obtaining a clear understanding of these systems. Modern echelle spectrographs and reticon readout devices allow radial velocity $\left(V_{R}\right)$ determinations to $\pm 1 / 2 \mathrm{~km} \mathrm{~s}^{-1}$, so observations over several years should allow the orbital $V_{R}$ curves to be measured. With these curves in hand, the size of the Roche lobe $\left(R_{L}\right)$ can be computed for a given total system mass and mass ratio(q). Optical and IR observations allow the spectral type and luminosity class of the giant to be determined (Kenyon and Gallagher 1983), and therefore one can determine whether a symbiotic is likely to be filling its Roche lobe.

\section{TECHNIQUE}

The echelle spectra which we have used to measure $V_{R}$ consist of a single, $\sim 40 \AA$ wide order centered at $\sim 5200 \AA$ with $\sim 0.2 \AA$ resolution. After emission lines are excised from the spectrum, $V_{R}$ is determined with a computerized cross-correlation technique (Tonry and Davis 1982). We have concentrated on S-type symbiotics with known photometric periods and which have not had a recent outburst, thereby ensuring that the $M$ giant photosphere is visible at $5200 \AA$. Once the $V_{R}$ are determined, orbital solutions are found using the Fourier decomposition technique described by Monet (1979). 


\section{RESULTS.}

TX CVn. The large quantity of data for this star allows an $11 \sigma$ level detection of the eccentricity $(e=0.2)$. The detection of a significant eccentricity in the orbit of TX CVn is surprising, as it is not expected on evolutionary grounds. For the remaining stars there is not enough data to justify fitting of an eccentric orbit, and we have set $e=0.0$. The orbital parameters derived for TX CVn are shown in Table 1, and the data and best fit eccentric orbit are plotted in Figure 1. Optical and IR observations of the giant in TX CVn indicate it is a M0 III with a radius $\left(R_{G}\right)$ of $\sim 35 \mathrm{R}_{\odot}$ (Kenyon and Gallagher 1983). If the mass of the giant is $\sim 1 \mathrm{M}_{\odot}$, and the mass of the hot star is $0.25-0.5 \mathrm{M}_{\odot}$, then the fraction of the Roche lobe filled, $R_{G} / R_{L} \sim 0.5$. The slightly larger giant radius required for the giant to fill its Roche lobe is most likely allowed by current observations.

EG And. The orbital solution in Table 1, which is based on data taken over 3.5 orbital cycles, confirms that of Oliversen et al. 1985. The phase of the radial velocity curve, when compared with the photometric phase for $H \alpha$ suggested by Oliversen et al., is within \pm 0.1 of that expected for an eclipse. The period determined from this radial velocity data is $5 \sigma$ larger than that derived by Smith (1980) from the $\mathrm{H} \alpha$ and photometric variations. While Smith does not quote and error in his derived 470 day period, examination of the data indicates it may be as large as 10 days, thus rendering the two periods nearly consistent. The IR and optical observations of Kenyon and Gallagher (1983) indicate that the giant component may have a radius of $\sim 100 \mathbf{R}_{\odot}$. Assuming the companion is a typical white dwarf with mass of $0.5 \mathrm{M}_{\odot}$, we find that $R_{L} / R_{G}<1.5$ for $q<3$, which indicates that the giant is likely to be filling its Roche lobe.

$\mathbf{Z}$ And. The orbital parameters deduced from these measurements (which cover two orbital cycles) are shown in Table 1, and the radial velocity curve is shown in Figure 1. The relative phasing of the radial velocity curve and photometric variations is a bit mysterious - spectroscopic conjunction occurs at $\phi=0.27 \pm 0.07$, where $\phi=0.0$ is defined as minimum light (Kenyon and Webbink 1984). This phasing is not that expected for (simple) reflection or eclipse phenomena, suggesting that rather complex geometric effects may be involved in producing the photometric variations. Optical and IR spectra indicate that the giant is an M3 II with a probable radius of $\sim 150 \mathbf{R}_{\odot}$ (Kenyon and Gallagher 1983). Given the orbital parameters in Table 1, the giant fills its Roche lobe if the mass of the accreting component $\left(M_{H}\right)$ is $1 \mathrm{M}_{\odot}$ and $q=1$, or if $M_{H}=0.5 \mathrm{M}_{\odot}$ and $q=2$. The giant may therefore be filling its Roche lobe.

UV Aur. These data cover 3 orbital cycles, but are sparse near phase 0.5 due to the proximity of the orbital period to 1 year. The orbital parameters derived from the radial velocity curve are shown in Table 1, and the data themselves are shown in Figure 1. Spectroscopic conjunction occurs at phase 0.4 of the ephemeris of Zakarov (1951), which defines phase zero to be at maximum light. Given the probable errors in the derived period, the relative phasings are consistent with that expected for eclipse or reflection phenomena. If the giant has a radius of $\sim 100 \mathrm{R}_{\odot}$ (Kenyon 1986), then it fills its Roche lobe for $M_{H} \sim 1 \mathrm{M}_{\odot}$ and $q \sim 1$. Assuming $M_{H}=1 \mathrm{M}_{\odot}$ and $q=2$, then $R_{L} / R_{G}=1.5$, so the giant in this system is likely to be filling its Roche lobe.

CI Cyg. The orbital parameters derived from these data (Table 1), which cover 2 full cycles, are substantially different from those reported by lijima (1982). Spectroscopic conjunction occurs at phase 0.98 on the ephemeris of Aller (1954), confirming the widespread 
belief (see Kenyon 1986 and references therein) that the photometric variations are caused by eclipses. The long duration of the eclipse in CI Cyg indicates that the giant, which has a radius of $\sim 175 \mathrm{R}_{\odot}$, fills its Roche lobe (Kenyon and Gallagher 1983). For $q=1$ to 2, the radial velocity data are consistent with a lobe filling giant with mass $\sim 1 \mathrm{M}_{\odot}$.

AG Dra. The orbital parameters derived from over 3 cycles of observations (see Figure 2) are shown in Table 1. Using the ephemeris and period found by Meinunger (1979), we find that spectroscopic conjunction occurs at $\phi=0.48 \pm 0.08$, as expected for eclipse or reflection effects (maximum light is defined to occur at $\phi=0.0$ ). The spectral type of the red star in AG Dra is somewhat uncertain as various authors have found spectral types ranging from G5 (Mirzoyan and Bartaya 1960) to K0 I (Huang 1983). The average seems to be about K3 III (Kenyon 1986), but the luminosity class is rather uncertain. Assuming a spectral type of $\mathrm{K} 3 \mathrm{III}$, the radius of the giant would be $\sim 30 \mathrm{R}_{\odot}$. For reasonable values of $q$ and system mass, the giant is well within its Roche lobe $\left(R_{L} / R_{G} \sim 4\right)$.

RW Hya. The orbital parameters derived from this more extensive data set (Table 1) tend to confirm the orbital parameters found earlier (Garcia 1986). Because the orbital period is so close to one year our phase coverage is rather poor (see Figure 2) even though we have observed $\sim 5$ orbital cycles. The phase of spectroscopic conjunction is $\phi=0.2 \pm 0.1$ on the ephemeris of Kenyon and Webbink (1984), which defines phase zero as maximum light. This relative phasing is rather difficult to understand, as it is nearly opposite that expected for reflection or eclipse phenomena. The giant in RW Hya is most likely a M2 III with a radius of $\sim 60 \mathrm{R}_{\odot}$ (Kenyon and Fernandez-Castro 1987). In this case the giant fills its Roche lobe only for $q \sim 1$ and $M_{G} \sim 0.2 \mathrm{M}_{\odot}$, which seems improbably low. Thus it appears that the giant substantially underfills its Roche lobe $\left(R_{L} / R_{G} \sim 2.0\right)$.

AG Peg. The orbital parameters found from these radial velocity measurements (Table 1) are very similar to those found by Hutchings, Cowley and Redman (1975). The data (which cover 1.3 orbital cycles) and best fit solution are shown in Figure 2. Using the ephemeris for minimum light found by Meinunger (1981) we find that spectroscopic conjunction occurs at phase $0.9 \pm 0.1$, consistent with that expected from eclipse or reflection effects. If the giant is a normal M3 III, as implied by the optical and IR observations (Kenyon and Gallagher 1983), then it must have a radius $<100 R_{\odot}$. The radial velocity measurements then require that the system have unreasonably small mass ( $M_{G} \sim 0.2 \mathrm{M}_{\odot}$, $q \sim 1$ ) if the giant is to fill its Roche lobe. We conclude that a giant with $R_{G}<100 \mathrm{R}_{\odot}$ will not fill its Roche lobe in this system.

AX Per. The orbital parameters derived from these data (which cover 2.4 cycles, see Figure 2) are shown in Table 1. The orbital period derived from these radial velocity measurements alone is $\sim 8 \sigma$ below the photometric period (Kenyon 1982). However, the photometric period is based on $\sim 600$ measurements covering 7 cycles, therefore must be considered more reliable. This view is further supported by the observation that the scatter in the radial velocities measured for AX Per about the fit curve is larger than that seen in other symbiotics (See Figures 1 and 2). Using the ephemeris for minimum light defined by Kenyon (1982), we find that spectroscopic conjunction occurs at phase $0.96 \pm 0.13$, i.e., consistent with that expected for eclipse or reflection effects. As the radius of the giant is somewhat unclear at present (see Kenyon and Gallagher 1983), we cannot determine whether it fills its Roche lobe or not. 
Table 1: Summary of Orbits from $V_{R}$ Measurements

\begin{tabular}{|c|l|l|l|l|r|r|r|}
\hline \hline Star & Best P (days) & Other P & $\mathrm{K}(\mathrm{km} / \mathrm{sec})$ & $\mathrm{e}$ & $\mathrm{f}(\mathrm{m})$ & $\gamma$ & $\mathrm{N}_{\text {points }}$ \\
\hline EG And & $492 \pm 4.3$ & $470 \pm 10$ & $7.1 \pm 0.2$ & 0.0 & .017 & -94 & 19 \\
Z And & $750 \pm 8$ & $756.85 \pm 1.0$ & $8.1 \pm 0.5$ & 0.0 & .042 & +2 & 19 \\
UV Aur & $388 \pm 5$ & $395.2 \pm ?$ & $5.1 \pm 0.3$ & 0.0 & .005 & +6 & 19 \\
TX CVn & $199.0 \pm 0.5$ & none & $6.1 \pm 0.1$ & 0.2 & .003 & +2 & 37 \\
Cr Cyg & $812 \pm 10$ & 855.25 & $6.5 \pm 0.3$ & 0.0 & .024 & +18 & 25 \\
AG Dra & $530 \pm 5$ & $554 \pm 5$ & $5.3 \pm 0.3$ & 0.0 & .009 & -146 & 17 \\
RW Hya & $366 \pm 2$ & $372.45 \pm 0.3$ & $8.7 \pm 0.9$ & 0.0 & .025 & +14 & 10 \\
AG Peg & $796 \pm 15$ & $827.0 \pm 4$ & $6.4 \pm 0.2$ & 0.0 & .022 & -14 & 17 \\
AX Per & $601 \pm 10$ & $681.6 \pm 7.2$ & $6.7 \pm 0.4$ & 0.0 & .021 & -114 & 19 \\
\hline
\end{tabular}

The $\gamma$ velocities are in $\mathrm{km} \mathrm{s}^{-1}$, and have errors of approximately $\pm 1 \mathrm{~km} \mathrm{~s}^{-1}$. The errors in the mass functions $f(m)=K^{3} P / 2 \pi G$ can be derived directly from the quoted errors on $P$ and $K$. The number of radial velocity measurements used in the orbital solution is $\mathrm{N}_{\text {points }}$.

\section{CONCLUSIONS.}

Continuing measurements of the $V_{R}$ in Symbiotics will allow more accurate determination of the orbital periods and perhaps the measurement of orbital eccentricities in more stars. Comparisons of the $V_{R}$ curve of the giant with those derived for emission lines should allow the structure of the emission line region to be mapped out. While the radial velocity curves allow the approximate size of the binary to be determined, the critical question of whether or not the giant fills its Roche lobe depends strongly upon the often uncertain nature of the giant. The IR and optical studies of Kenyon and Gallagher (1983) go a long way toward solving this problem, but clearly there is more work to be done.

REFERENCES.

Aller, L.H., Publ. DAO Victoria, B.C., 9, 321.

Garcia, M.R., 1986, Astr. Ap. 91, 1400.

Huang, C.C., 1983, in IAU Colloquium No. 70, The Nature of Symbiotic Stars, ed. M. Friedjung and R. Viotti, (Dordrecht:Reidel), p. 151.

Hutchings, J.B., Cowley, A.P., and Redman, R.O., 1975 Ap.J. 201, 404.

lijima, T., 1982 Astr. Ap. 116, 210.

Kenyon, S.J., and Fernandez-Castro, T., 1987 Ap.J. 316, 427.

Kenyon, S.J., 1986, The Symbiotic Stars, (Cambridge: Cambridge University Press).

Kenyon, S.J., and Webbink, R.F., 1984, Ap.J. 279, 252.

Kenyon, S.J., and Gallagher, J.S., 1983 A.J., 88, 666.

Kenyon, S.J., 1982 P.A.S.P. 94, 165.

Mirzoyan, L.V., and Bartaya, R.A., 1960, Izv. Abastumani Astrofiz. Obs., No. 25, 121.

Meinunger, L., 1981, Inf. Bull. Var. Stars, No. 2016.

Meinunger, L., 1979 Inf. Bull. Var. Stars, No. 1611.

Monet, D.G., 1979 Ap.J. 234, 275.

Oliversen, N.A., Anderson, C.M., Stencel, R.E., and Slovak, M.H., 1985 A p.J. 295, 620.

Patterson, J., 1984 Ap.J. Suppl. 54, 443.

Payne-Gaposchkin, C., 1957, The Galactic Novae (Amsterdam: North Holland). 

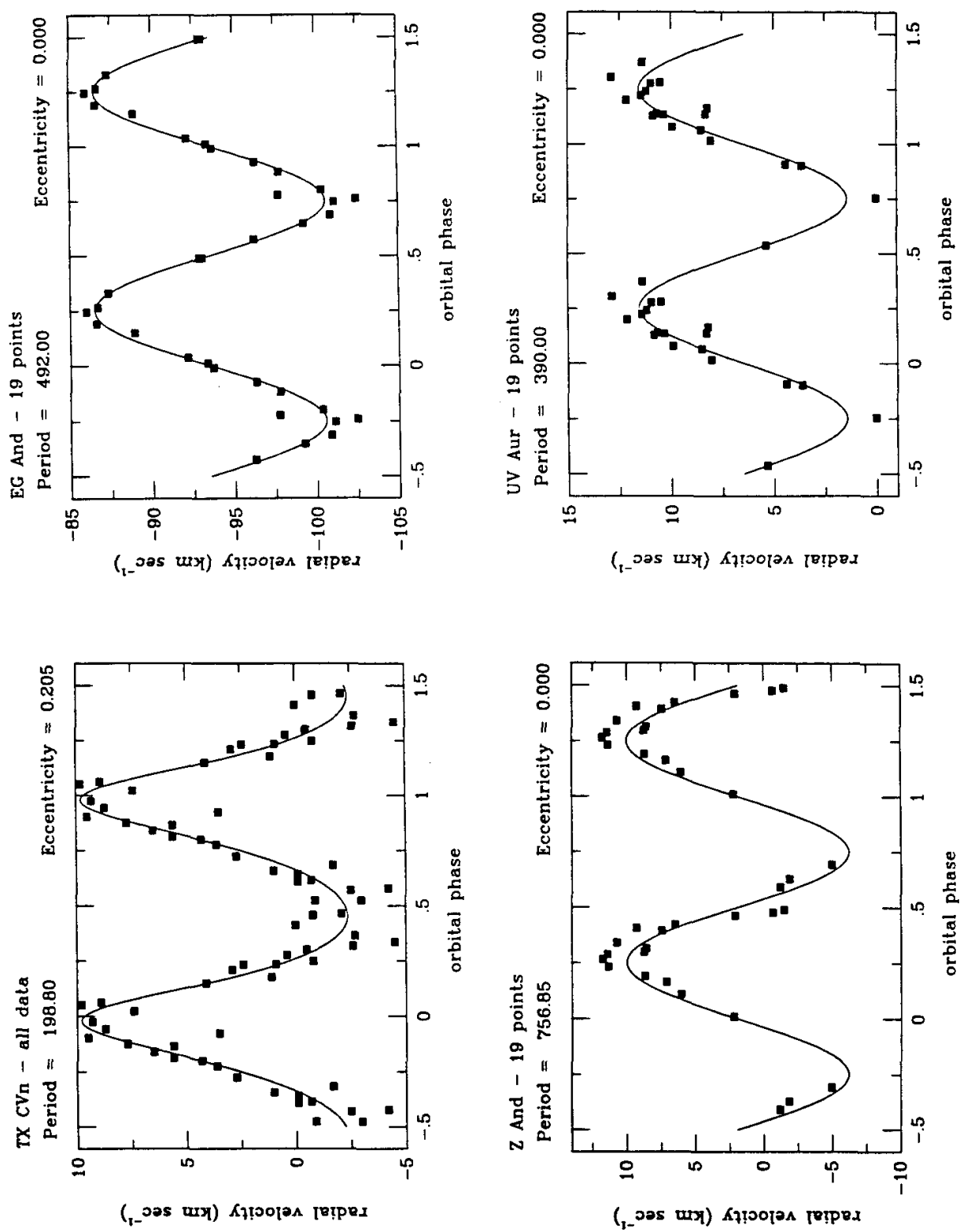

Figure 1: Radial Velocity Curves of Symbiotic Stars

The measured radial velocities and orbital solutions based on photometric periods are shown for TX CVn, EG And, Z And, and UV Aur. 

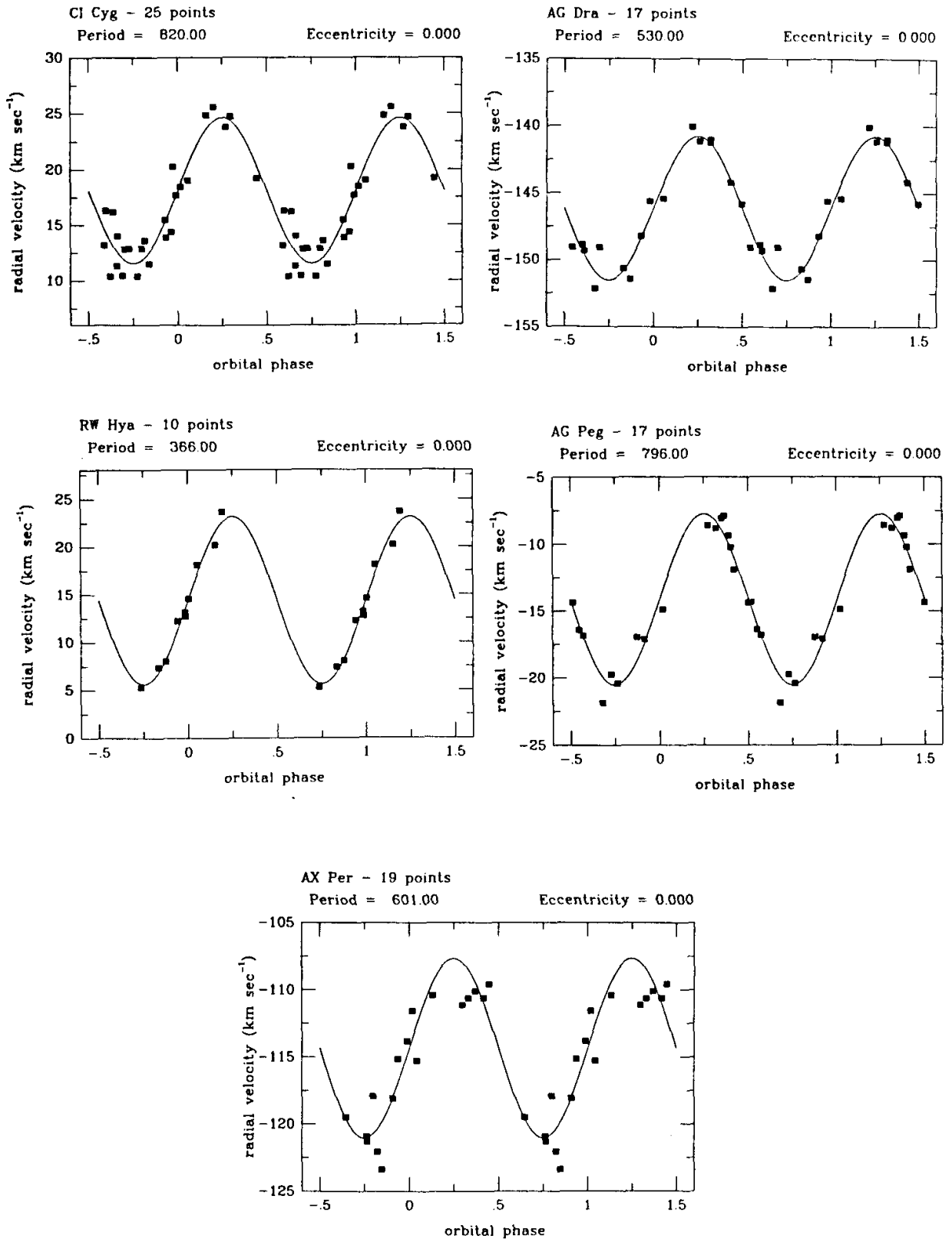

Figure 2: Radial Velocity Curves of Symbiotic Stars

The measured radial velocities and orbital solutions based on photometric perids are shown for CI Cyg, AG Dra, RW Hya, AG Peg, and AX Per. 